\title{
Review
}

\section{Monocytes in Aging and Exercise}

\author{
Johnathan R. Yarbro ${ }^{1}$, Brandt D. Pence ${ }^{1,2, *(i)}$ \\ ${ }^{1}$ School of Health Studies, University of Memphis, Memphis, TN, USA \\ ${ }^{2}$ Center for Nutraceutical and Dietary Supplement Research, University of Memphis, Memphis, TN, USA
}

\section{Article Information \\ History: \\ Received: August 17, 2018 \\ Accepted: October 13, 2018 \\ Published: October 31, 2018}

\section{Keywords:}

Exercise immunology

Immunosenescence

Immunogerontology

Older adults

Physical activity

\section{ABSTRACT}

Monocytes are cells of the innate immune system which perform important functions, including inflammatory cytokine production and phagocytosis, and participate in pathogen defense. In aging and disease, monocyte functions are dysregulated, and this dysregulation has been conclusively linked to the pathogenesis of a variety of age-related chronic diseases. In this review, we summarize the current literature supporting exercise training as a potential therapy for improving monocyte function, with particular attention to studies in the aging population. Substantial evidence suggests exercise is an effective therapy for modulating monocyte biology, although mechanisms by which this occurs are still not well understood.

\section{Aging}

The percentage of Americans over the age of 65 is expected to increase to nearly $20 \%$ by 2030 [1]. This change will have significant effects on the economy, healthcare, and society in general. Aging is the highest risk factor for the majority of chronic diseases - including cardiovascular disease, diabetes, arthritis, and cancer [2]. While lifespan continues to rise, healthspan (the length of time someone is healthy) has increased more slowly and Americans are living longer with impaired health and disabilities [3]. Interventions are needed to improve the health and quality of life of the aging population, and studies show that the compression of morbidity is possible with lifestyle changes, pharmaceuticals, and continuous medical advances [2].

\section{Inflammaging}

Aging is associated with chronic, low-level, systemic inflammation (termed inflammaging) that contributes to most, if

*Correspondence: Brandt D. Pence, Center for Nutraceutical and Dietary Supplement Research, University of Memphis, 304 Elma Roane Fieldhouse, 495 Zach H. Curlin St., Memphis, TN 38152.

E-mail address: bdpence@memphis.edu not all, age-related diseases [4]. Older adults have higher serum levels of several pro-inflammatory cytokines/proteins such as IL-6, IL-1ß, CRP, and TNF- $\alpha$. Elevated levels of these molecules in circulation, most notably IL-6, are correlated with an increased risk of morbidity and mortality in elderly populations [5]. Furthermore, they are associated with sarcopenia, malnutrition, reduced bone density, diabetes, arthritis, atherosclerosis, cognitive decline, and other diseases of aging [6]. There is no consensus on the causes of inflammaging, though it's likely due to a host of factors that become dysregulated with age. These "hallmarks of aging" include reduced autophagy and mitophagy, accumulation of DNA and mtDNA damage leading to genomic instability, epigenetic changes, telomere shortening, cellular and immune senescence, dysbiosis, chronic antigenic stress, diminished proteostasis, altered metabolic signaling, stem cell exhaustion, increased cellular garbage, and mitochondrial dysfunction [7]. Specific details of the aging process and inflammaging are beyond the scope of this article and we recommend a comprehensive review article for more information [7]. In this review article we focus on monocytes and their roles in diseases, aging, and exercise.

\section{Monocytes}

Monocytes are circulating mononuclear phagocytes of the terms of the Creative Commons Attribution License (http://creativecommons.org/licenses/by/4.0/), which permits unrestricted use, distribution, and reproduction in any medium, provided the original work is properly cited. 
innate immune system and have several functions including phagocytosis, antigen presentation, and the secretion of anti- and pro-inflammatory cytokines [8]. Upon infection or tissue damage, they are extravasated into tissue and differentiate into macrophages or dendritic cells [9]. Monocytes have been divided into three subtypes based on their expression of the cell surface markers CD14 (a co-receptor with TLR4 for the detection of LPS), and CD16 (a FC $\gamma$ III receptor that mediates antibody-dependent cell-mediated cytotoxicity) [10]. Classical Monocytes $\left(\mathrm{CD} 14^{+} \mathrm{CD} 16^{-}\right)$have high levels of CD14 on their surface, little to no expression of CD16, and are the most plentiful type ( 80-90\%). Non-classical $\left(\mathrm{CD} 14^{\mathrm{dim}} \mathrm{CD} 16^{+}\right)$and intermediate monocytes $\left(\mathrm{CD} 14^{+} \mathrm{CD} 16^{+}\right)$both express $\mathrm{CD} 16$, although non-classical monocytes have very low expression of CD14 whereas intermediate monocytes have high expression of CD14 [10]. It is important to note that prior to 2010, monocytes were primarily categorized into classical $\left(\mathrm{CD} 14^{+} \mathrm{CD} 16^{-}\right)$and inflammatory $\left(\mathrm{CD} 14^{+} \mathrm{CD} 16^{+}\right)$subtypes, and many manuscripts which will be discussed in the following sections used this classification.

In general, classical monocytes have been shown to be primarily involved in tissue repair, immune responses, migration to inflamed tissues, and phagocytosis [11]. They display the broadest range of cytokines in response to LPS, express the highest levels of genes involved in pattern recognition and phagocytosis (such as CD93, CD36, CD209, and CD163), exhibit high production of reactive oxygen species, and express high levels of chemotaxis genes CCR2, CXCR1, and CXCR2 [12]. During an inflammatory response CCL2 (MCP-1), which binds and activates CCR2, is released from the bone marrow and promotes classical monocyte release into circulation [13]. Once in circulation the classical monocytes make their way to the site of inflammation and release pro-inflammatory cytokines such as IL-6, TNF-a, and IL-1ß [14]. During steady-state, classical monocytes have a very short circulating lifespan ( $\sim$ day), and approximately $1 \%$ transition into intermediate monocytes ( $\sim 4$ day lifespan), of which most eventually transition into non-classical monocytes ( $\sim$ day lifespan) [15].

Intermediate monocytes are thought to be a transitional population between the classical and non-classical subsets. Due to their diversity, intermediate monocytes have a wide range of functions and can display characteristics of both classical and non-classical monocytes. They are primarily involved in antigen presentation, phagocytosis, and parasite recognition $[16,17]$. Additionally, they have generally been shown to be the most pro-inflammatory subset, producing high amounts of TNF- $\alpha$ and IL-1ß. Furthermore, they are proportionally increased with age and in many chronic diseases [18].

Non-classical monocytes have high expression of the adhesion-related receptor CX3CR1 and display patrolling behavior within the vasculature, removing debris, rearranging the cytoskeleton, preventing tumor metastasis, and resolving inflammation [12]. Like intermediate monocytes they are also increased with age [18]. Recently, they were shown to display more signs of senescence than intermediate or classical monocytes, as they express the highest levels of NFKB, IL-1a, and miR-146a, and have the shortest telomeres, all of which are associated with a senescence-associated secretory phenotype [19].

\section{Monocytes and Aging}

Although aging is multi-factorial, monocytes may very well play an essential role in aging pathology. Their heterogenous and highly adaptable nature, ability to respond to pathogens and cellular garbage, communication with the adaptive immune system, and numerous defects with age make them a key contributor to inflammaging. This section will briefly describe how the inflammaging environment can affect monocyte phenotype and functions, for a more detailed description of all the changes that occur to monocytes with age we recommend this review article [20].

Older adults have higher circulating levels of mitochondrial DNA (mtDNA), IL-6, TNF- $\alpha$, and other proinflammatory factors that dysregulate monocyte functions, which can in turn further progress inflammaging. For instance, $\mathrm{mtD}$ NA, which can act as a DAMP and activate monocytes via TLR-9, is increased with age and is positively correlated with TNF- $\alpha$ and IL- 6 in the blood [21]. In vitro stimulation of monocytes from young adults with LPS and high mtDNA concentrations, similar to levels found in older adults, increased TNF- $\alpha$ production higher than by LPS alone [21]. Given that LPS is also elevated systemically with age, probably due to dysbiosis [22], microenvironment changes are likely to be linked to dysregulated monocyte function and to contribute to the inflammaging environment.

Higher circulating TNF- $\alpha$ levels with age have been shown to cause multiple effects in monocyte phenotypes as well. TNF- $\alpha$ has been shown to induce the M1 ("pro-inflammatory") phenotype in macrophages, and macrophage populations are skewed towards the M1 phenotype with age [23]. Additionally, TNF- $\alpha$ may skew monocytes towards a more pro-inflammatory phenotype, as systemic TNF- $\alpha$ is positively correlated with intermediate monocyte frequency, and aged TNF- $\alpha$ knock-out mice had similar levels of circulating pro-inflammatory monocytes as younger mice [24]. CD11b (ITGAM), an integrin involved in transendothelial migration and associated with atherosclerotic plaque formation, is increased in classical and non-classical monocytes with age [18]. While it's unknown if TNF- $\alpha$ is primarily responsible for this altered expression in monocytes, it has been shown to increase CD11b expression in neutrophils in vivo and ex vivo, and CD11b+ neutrophils are positively correlated with plasma TNF- $\alpha$ [25].

\section{Exercise}

Given the above evidence suggesting that monocytes are important mediators of inflammatory and pathogen responses, and further that monocyte functions are dysregulated in aging and disease, therapies to combat monocyte dysfunc- 
tion are of particular importance. Physical exercise has been known for decades to be highly beneficial for overall health. A variety of epidemiological and experimental studies have described the vast benefits of physical activity, and a full treatment of this literature is not possible here. For a broad overview of the topic, we refer readers to a recent review article covering the beneficial effects of exercise on preventing or treating a variety of age-related conditions [26].

\section{Exercise and Immunity}

The impact of exercise on immune function has been widely investigated since the mid-1980s. In the field of exercise immunology, an important distinction is often made between acute, or single bouts of exercise, compared to chronic exercise training. These modes can have disparate and often opposite effects on immunity, and these effects are also dependent on intensity and duration of both acute exercise bouts and chronic exercise training [27]. One of the earliest observations in the field of exercise immunology was of acute exercise-induced leukocytosis, an increase in the number of circulating white blood cells. Indeed, this leukocytosis was described as early as 1893 [28]. Much more recently, chronic exercise has been investigated as an anti-inflammatory intervention to treat or prevent inflammation-related chronic diseases [27]. Monocytes have been heavily investigated in both contexts, and Figure 1 summarizes the current consensus as to the differential effects of acute and chronic exercise on monocytes (Figure 1). Given space limitations, we will focus this review on the effects of chronic exercise, with special attention to studies examining monocytes in the aging context. For additional information on acute exercise, we refer the reader to a comprehensive review which, although now several years old, outlines the basics of the acute monocyte response to exercise [27].

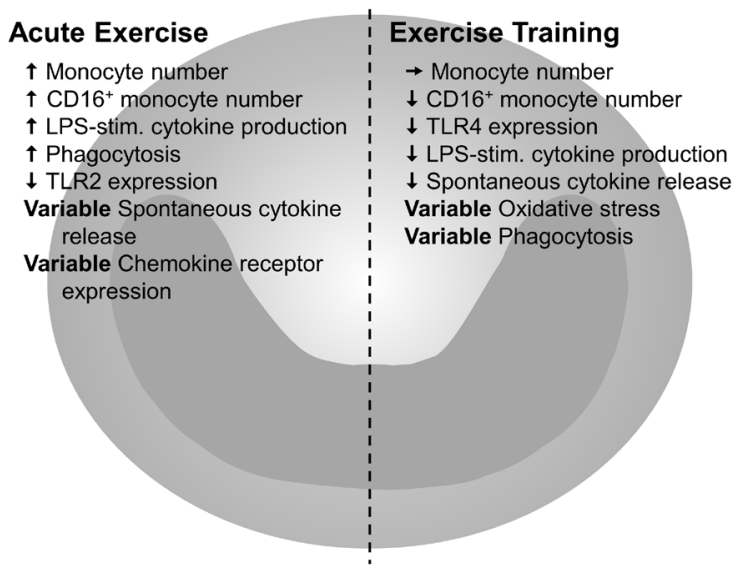

Figure 1. Well-supported effects of acute and chronic exercise on monocytes.

$\uparrow$ exercise increases effect, $\downarrow$ exercise decreases effect, $\rightarrow$ no change due to exercise.

\section{Exercise and Monocyte Subpopulations}

Although many exercise studies have focused on the effects of acute exercise on monocyte phenotype and function, some studies have examined the effect of exercise training and how it can induce sustained or persistent changes in monocytes. Given the association between CD16+ monocyte subtypes and a variety of inflammatory diseases [2931], the ability of exercise to alter subpopulation frequencies has naturally been of interest. A primary target for intervention has been the elderly, due to their higher frequency of CD16+ monocyte subpopulations [18] and their propensity to develop chronic inflammatory diseases [32-34]. Although earlier work by Woods et al. demonstrated that a 6 month exercise intervention in sedentary older adults did not alter total circulating monocyte numbers [35], an important paper by Timmerman et al. later demonstrated that exercise training in older adults decreased the proportion of $\mathrm{CD}^{+} 6^{+}$monocytes [36] (note this was prior to the establishment of the intermediate and non-classical CD16 ${ }^{+}$subpopulations). Several later works by the same group, still using the now-outdated "inflammatory" CD16 $6^{+}$monocyte definition, showed similar results in both endurance and resistant training [37-39]. To date, the effect of exercise training on monocyte populations using the modern definition has not been widely investigated, although it was recently demonstrated that exercise training reduced intermediate but not non-classical monocyte proportion in hemodialysis patients [40].

\section{Exercise and Monocyte Receptor Expression}

Exercise training has been somewhat more widely investigated for its effect on TLR expression, which is often used as a proxy for inflammation. In an aged population, exercise training reduced whole blood mRNA expression of TLR4 and of pan-monocyte marker CD14 [41]. Follow-up studies supported this, demonstrating reduced TLR4 protein expression on isolated leukocytes [42] and then on CD14 ${ }^{+}$ monocytes [43] after resistance or combined exercise training respectively in older adults. McFarlin et al. continued this line of inquiry by demonstrating that exercise training reduced TLR4 protein expression on circulating leukocytes independent of subject age [44], and Timmerman et al. found that previously-active older adults had reduced TLR4 protein expression on classical (CD16) monocytes compared to sedentary controls [36]. Additionally, both high intensity interval training and moderate intensity continuous exercise training were shown to reduce TLR4 but not TLR2 expression on blood monocytes [45]. Although the important characteristics of exercise training which underlie the control of monocyte receptor expression have not been elucidated, a study in obese mice suggests that the exercise training effect on monocyte TLR expression may be duration-dependent [46]. 
Exercise and Monocyte Function

As with acute exercise, the effect of exercise training on monocyte function has been a relatively active area of study. Results have been mixed depending on mode of exercise and subject population. For example, Nieman et al. found no effect of a 12-week walking intervention on phagocytosis or oxidative burst in monocytes in middle-aged obese women [47], while 10 weeks of either moderate exercise or high-intensity interval training in healthy sedentary adults improved the same parameters [48]. Voluntary wheel running in adult Balb/c mice has additionally been shown to have no effect on monocyte phagocytosis [49]. These differences may be attributable to differences in study population and/ or mode or intensity of exercise. Several studies have also examined the effect of exercise on chemotactic properties of monocytes, with a diet + exercise intervention reducing monocyte adhesion and chemotactic activity in obese men [50] and a 3 week exercise intervention reducing monocyte adhesion in a similar study [51].

Multiple studies have demonstrated an exercise training-induced reduction in inflammatory cytokine production in resting and stimulated monocytes. Timmerman et al. found reduced LPS-stimulated TNFa production after 12 weeks of exercise training in older adults [36], similar to that found with high-intensity training in younger individuals [52]. Resistance training has likewise been shown to reduce TNFa, IL- $1 \beta$, and IL- 6 production in whole blood in older women [53]. In unstimulated monocytes, swimming exercise reduced spontaneous IL-1 $\beta$, IL- 6 , and TNF $\alpha$ release and increased release of anti-inflammatory cytokine IL-10 in fibromyalgia patients [54]. Similarly, a two-month aerobic exercise intervention reduced expression of genes related to inflammation and oxidative stress [55].

Finally, the field of immunometabolism (the study of the contribution of metabolic processes to immune cell function) has exploded in recent years [56]. Metabolic processes, especially with respect to mitochondrial metabolism, are known to be dysregulated during the aging process in a variety of tissues and cell types [7]. Indeed, in support of this we have recently demonstrated that aging impairs mitochondrial respiratory capacity in human monocytes [57]. Exercise has been shown to increase mitochondrial respiration in peripheral blood mononuclear cells [58] and thus is a potential mechanism by which exercise alters monocyte function in older adults. To date, this is speculative and bears further investigation.

\section{Summary}

A number of studies have examined monocyte phenotype and function in the context of a variety of exercise paradigms. To date several of these studies have focused on changes to monocytes as a result of consistent physical activity. Many of these have focused on aged populations, and exercise training-induced reductions in $\mathrm{CD}^{+} 6^{+}$monocyte populations and in LPS-stimulated cytokine release are now fairly well-supported. Reduced pattern recognition receptor expression, especially TLR4, on monocytes with exercise training also has convincing evidential support. The mechanisms by which exercise training mediate these changes are currently unknown, but many effects appear independent of diet or weight loss. Future research will be critical to determine the mechanism by which exercise training acts to alter monocyte phenotype and inflammation, which should allow for exercise therapies which are targeted to these responses to be developed. Additionally, few studies have compared multiple modes or intensities of exercise for their impact on monocyte phenotype or function. Therefore, although both aerobic and resistance exercise appear to be efficacious in modulating monocyte biology, future studies which shed light on this important variable will be important for exercise prescription purposes.

\section{Conflicts of Interest}

The authors declare no conflict of interest.

\section{REFERENCES}

1. Anderson LA, Goodman RA, Holtzman D, Posner SF, Northridge ME. Aging in the united states: Opportunities and challenges for public health. Am J Public Health. 2012; 102 (3):393-395.

2. Kennedy BK, Berger SL, Brunet A, et al. Geroscience: Linking aging to chronic disease. Cell. 2014; 159 (4):709-713.

3. Chatterji S, Byles J, Cutler D, Seeman T, Verdes E. Health, functioning, and disability in older adults-present status and future implications. Lancet. 2015; 385 (9967):563-575.

4. Franceschi C, Campisi J. Chronic inflammation (inflammaging) and its potential contribution to age-associated diseases. J Gerontol A Biol Sci Med Sci. 2014; 69(S1):S4-9.

5. Franceschi C, Garagnani P, Vitale G, Capri M, Salvioli S. Inflammaging and 'garb-aging'. Trends Endocrinol Metab. 2017; 28(3):199-212.

6. Michaud M, Balardy L, Moulis G, et al. Proinflammatory cytokines, aging, and age-related diseases. J Am Med Dir Assoc. 2013; 14 (12):877-882.

7. Lopez-Otin C, Blasco MA, Partridge L, Serrano M, Kroemer G. The hallmarks of aging. Cell. 2013; 153 (6):1194-1217.

8. Medvedev AE, Sabroe I, Hasday JD, Vogel SN. Tolerance to microbial tlr ligands: Molecular mechanisms and relevance to disease. J Endotoxin Res. 2006; 12(3):133-150.

9. Yang J, Zhang L, Yu C, Yang XF, Wang H. Monocyte and macrophage differentiation: Circulation inflammatory monocyte as biomarker for inflammatory diseases. Biomark Res. 2014; 2(1):1.

10. Ziegler-Heitbrock L, Hofer TP. Toward a refined definition of monocyte subsets. Front Immunol. 2013; 4:23. 
11. Mukherjee R, Kanti Barman P, Kumar Thatoi P, Tripathy R, Kumar Das B, Ravindran B. Non-classical monocytes display inflammatory features: Validation in sepsis and systemic lupus erythematous. Sci Rep. 2015; 5:13886.

12. Wong KL, Tai JJ, Wong WC, et al. Gene expression profiling reveals the defining features of the classical, intermediate, and nonclassical human monocyte subsets. Blood. 2011; 118(5):e16-31.

13. Serbina NV, Pamer EG. Monocyte emigration from bone marrow during bacterial infection requires signals mediated by chemokine receptor ccr2. Nat Immunol. 2006; 7(3):311-317.

14. Boyette LB, Macedo C, Hadi K, et al. Phenotype, function, and differentiation potential of human monocyte subsets. PLoS One. 2017; 12(4):e0176460.

15. Patel AA, Zhang Y, Fullerton JN, et al. The fate and lifespan of human monocyte subsets in steady state and systemic inflammation. J Exp Med. 2017; 214(7):19131923.

16. Gren ST, Rasmussen TB, Janciauskiene S, Hakansson K, Gerwien JG, Grip O. A single-cell gene-expression profile reveals inter-cellular heterogeneity within human monocyte subsets. PLoS One. 2015; 10(12):e0144351.

17. Turner JD, Bourke CD, Meurs L, et al. Circulating cd14brightcd16+ 'intermediate' monocytes exhibit enhanced parasite pattern recognition in human helminth infection. PLoS Negl Trop Dis. 2014; 8(4):e2817.

18. Hearps AC, Martin GE, Angelovich TA, et al. Aging is associated with chronic innate immune activation and dysregulation of monocyte phenotype and function. Aging Cell. 2012; 11(5):867-875.

19. Ong SM, Hadadi E, Dang TM, et al. The pro-inflammatory phenotype of the human non-classical monocyte subset is attributed to senescence. Cell Death Dis. 2018; 9(3):266

20. Albright JM, Dunn RC, Shults JA, Boe DM, Afshar M, Kovacs EJ. Advanced age alters monocyte and macrophage responses. Antioxid Redox Signal. 2016; 25(15):805-815.

21. Pinti M, Cevenini E, Nasi M, et al. Circulating mitochondrial DNA increases with age and is a familiar trait: Implications for "inflamm-aging". Eur J Immunol. 2014; 44 (5):1552-1562.

22. Kim KA, Jeong JJ, Yoo SY, Kim DH. Gut microbiota lipopolysaccharide accelerates inflamm-aging in mice. BMC Microbiol. 2016; 16:9.

23. Linton PJ, Thoman ML. Immunosenescence in monocytes, macrophages, and dendritic cells: Lessons learned from the lung and heart. Immunol Lett. 2014; 162 (1 Pt B):290-297.

24. Puchta A, Naidoo A, Verschoor CP, et al. Tnf drives monocyte dysfunction with age and results in impaired anti-pneumococcal immunity. PLoS Pathog. 2016; 12(1):e1005368.

25. Verschoor CP, Loukov D, Naidoo A, et al. Circulating tnf and mitochondrial DNA are major determinants of neutrophil phenotype in the advanced-age, frail elderly.
Mol Immunol. 2015; 65(1):148-156.

26. Pedersen BK, Saltin B. Exercise as medicine - evidence for prescribing exercise as therapy in 26 different chronic diseases. Scand J Med Sci Sports. 2015; 25 (S3)1-72.

27. Walsh NP, Gleeson M, Shephard RJ, et al. Position statement. Part one: Immune function and exercise. Exerc Immunol Rev. 2011; 17:6-63.

28. Shephard RJ. Development of the discipline of exercise immunology. Exerc Immunol Rev. 2010; 16:194-222.

29. Shantsila E, Wrigley B, Tapp L, et al. Immunophenotypic characterization of human monocyte subsets: Possible implications for cardiovascular disease pathophysiology. J Thromb Haemost. 2011; 9(5):1056-1066.

30. Grip O, Bredberg A, Lindgren S, Henriksson G. Increased subpopulations of cd16(+) and cd56(+) blood monocytes in patients with active crohn's disease. Inflamm Bowel Dis. 2007; 13(5):566-572.

31. Tsukamoto M, Seta N, Yoshimoto K, Suzuki K, Yamaoka K, Takeuchi T. Cd14(bright)cd16+ intermediate monocytes are induced by interleukin-10 and positively correlate with disease activity in rheumatoid arthritis. Arthritis Res Ther. 2017; 19(1):28.

32. North BJ, Sinclair DA. The intersection between aging and cardiovascular disease. Circ Res. 2012; 110(8):10971108.

33. Finkel T, Serrano M, Blasco MA. The common biology of cancer and ageing. Nature. 2007; 448(7155):767-774.

34. Kalyani RR, Egan JM. Diabetes and altered glucose metabolism with aging. Endocrinol Metab Clin North Am. 2013; 42(2):333-347.

35. Woods JA, Ceddia MA, Wolters BW, Evans JK, Lu Q, McAuley E. Effects of 6 months of moderate aerobic exercise training on immune function in the elderly. Mech Ageing Dev. 1999; 109(1):1-19.

36. Timmerman KL, Flynn MG, Coen PM, Markofski MM, Pence BD. Exercise training-induced lowering of inflammatory $(\mathrm{cd} 14+\mathrm{cd} 16+)$ monocytes: A role in the anti-inflammatory influence of exercise? J Leukoc Biol. 2008; 84(5):1271-1278.

37. Markofski MM, Flynn MG, Carrillo AE, Armstrong CL, Campbell WW, Sedlock DA. Resistance exercise training-induced decrease in circulating inflammatory cd14+cd16+ monocyte percentage without weight loss in older adults. Eur J Appl Physiol. 2014; 114(8):17371748.

38. Markofski MM, Carrillo AE, Timmerman KL, et al. Exercise training modifies ghrelin and adiponectin concentrations and is related to inflammation in older adults. J Gerontol A Biol Sci Med Sci. 2014; 69(6):675681.

39. Coen PM, Flynn MG, Markofski MM, Pence BD, Hannemann RE. Adding exercise to rosuvastatin treatment: Influence on c-reactive protein, monocyte toll-like receptor 4 expression, and inflammatory monocyte $(\mathrm{cd} 14+\mathrm{cd} 16+)$ population. Metabolism. 2010; 59(12):1775-1783.

40. Dungey M, Young HML, Churchward DR, Burton JO, 
Smith AC, Bishop NC. Regular exercise during haemodialysis promotes an anti-inflammatory leucocyte profile. Clin Kidney J. 2017; 10(6):813-821.

41. Flynn MG, McFarlin BK, Phillips MD, Stewart LK, Timmerman KL. Toll-like receptor 4 and cd14 mrna expression are lower in resistive exercise-trained elderly women. J Appl Physiol (1985). 2003; 95(5):1833-1842.

42. McFarlin BK, Flynn MG, Campbell WW, Stewart LK, Timmerman KL. Tlr4 is lower in resistance-trained older women and related to inflammatory cytokines. Med Sci Sports Exerc. 2004; 36(11):1876-1883.

43. Stewart LK, Flynn MG, Campbell WW, et al. Influence of exercise training and age on cd14+ cell-surface expression of toll-like receptor 2 and 4 . Brain Behav Immun. 2005; 19(5):389-397.

44. McFarlin BK, Flynn MG, Campbell WW, et al. Physical activity status, but not age, influences inflammatory biomarkers and toll-like receptor 4. J Gerontol A Biol Sci Med Sci. 2006; 61(4):388-393.

45. Robinson E, Durrer C, Simtchouk S, et al. Short-term high-intensity interval and moderate-intensity continuous training reduce leukocyte tlr4 in inactive adults at elevated risk of type 2 diabetes. J Appl Physiol (1985). 2015; 119(5):508-516.

46. Carpenter KC, Strohacker K, Breslin WL, Lowder TW, Agha NH, McFarlin BK. Effects of exercise on weight loss and monocytes in obese mice. Comp Med. 2012; 62(1):21-26.

47. Nieman DC, Nehlsen-Cannarella SL, Henson DA, et al. Immune response to exercise training and/or energy restriction in obese women. Med Sci Sports Exerc. 1998; 30(5):679-686.

48. Bartlett DB, Shepherd SO, Wilson OJ, et al. Neutrophil and monocyte bactericidal responses to 10 weeks of low-volume high-intensity interval or moderate-intensity continuous training in sedentary adults. Oxid Med Cell Longev. 2017; 2017:8148742.

49. van de Weert-van Leeuwen PB, de Vrankrijker AM, Fentz J, et al. Effect of long-term voluntary exercise wheel running on susceptibility to bacterial pulmonary infections in a mouse model. PLoS One. 2013; 8(12):e82869.

50. Roberts CK, Won D, Pruthi S, et al. Effect of a shortterm diet and exercise intervention on oxidative stress, inflammation, mmp-9, and monocyte chemotactic activity in men with metabolic syndrome factors. J Appl Physiol (1985). 2006; 100(5):1657-1665.

51. Roberts CK, Won D, Pruthi S, Lin SS, Barnard RJ. Effect of a diet and exercise intervention on oxidative stress, inflammation and monocyte adhesion in diabetic men. Diabetes Res Clin Pract. 2006; 73(3):249-259.

52. Sloan RP, Shapiro PA, Demeersman RE, et al. Aerobic exercise attenuates inducible tnf production in humans. J Appl Physiol (1985). 2007; 103(3):1007-1011.

53. Phillips MD, Flynn MG, McFarlin BK, Stewart LK, Timmerman KL. Resistance training at eight-repetition maximum reduces the inflammatory milieu in elderly women. Med Sci Sports Exerc. 2010; 42(2):314-325.

54. Ortega E, Bote ME, Giraldo E, Garcia JJ. Aquatic exercise improves the monocyte pro- and anti-inflammatory cytokine production balance in fibromyalgia patients. Scand J Med Sci Sports. 2012; 22(1):104-112.

55. Gano LB, Donato AJ, Pierce GL, et al. Increased proinflammatory and oxidant gene expression in circulating mononuclear cells in older adults: Amelioration by habitual exercise. Physiol Genomics. 2011; 43(14):895902.

56. O'Neill LA, Kishton RJ, Rathmell J. A guide to immunometabolism for immunologists. Nat Rev Immunol. 2016; 16(9):553-565.

57. Pence BD, Yarbro JR. Aging impairs mitochondrial respiratory capacity in classical monocytes. Exp Gerontol. 2018; 108:112-117.

58. Kocher M, McDermott M, Lindsey R, et al. Short communication: Hiv patient systemic mitochondrial respiration improves with exercise. AIDS Res Hum Retroviruses. 2017; 33(10):1035-1037. 